\title{
CONSTRUCTION OF ISOLOGY FUNCTORS
}

\author{
BY \\ ARTHUR H. COPELAND, JR.(1)
}

This paper is, hopefully, a step in the direction of obtaining a complete system of algebraic invariants for the isotopy classes of finitely triangulable spaces. We construct a large family of isotopy functors defined on a category of embeddings of topological pairs and having values in the category of homomorphisms of graded abelian groups. These functors are formally like homotopy and homology functors, having a boundary operator and satisfying an exactness axiom, and some of them are especially well behaved: they are isology functors, which is to say, they satisfy an excision axiom. These functors are defined in the first three sections and their basic properties are established.

The rest of the paper is concerned with testing whether these functors can tell one space from another. Ideally, one should construct a functor $T$ with the property that if $f$ is an embedding and if $T(f)$ is an isomorphism, then $f$ is an isotopy equivalence. In fact, somewhat less than this has happened. The ideal situation holds for embeddings of 1-dimensional finitely triangulable spaces, but at the next level of complexity, embeddings of 2-dimensional finitely triangulable cone spaces, there are nonequivalences which induce isomorphisms. This gap may be partially closed by employing the deleted product functor, but there still remain some spaces which look alike to all of the functors that the author knows how to deal with gracefully. However, it should be noted that the remaining cone spaces are quite simple and it seems unlikely that they will resist algebraic analysis for long.

1. The basic functors. If $X$ is a topological space, let $c X=X^{*} p$ be the join of $X$ with a point $p=a X$ not in $X$. The pair $(c X, a X)$ is called a cone over $X, c X$ is the space of the cone and $a X$ is the apex. The space $X$ is naturally embedded in $c X$ and is called the base of the cone. A map $f: X \rightarrow Y$ induces a map $c f: c X, a X \rightarrow c Y$, $a Y$ such that each segment from $x \in X$ to $a X$ is mapped linearly onto the segment from $f(x)$ to $a Y$. Any two cones over the same space are topologically equivalent pairs, and we choose to ignore the difference between them. In this spirit, we demand that if $Y \subset X$, then $a Y=a X$ and $c Y \subset c X$, so that $c$ is now regarded as a covariant functor from maps between topological pairs to maps between pairs with basepoint. If $f$ is an embedding, then $c f$ is also an embedding, and if $f$ is isotopic to $g$, then $c f$ is isotopic to $c g$; the second isotopy leaves the apex fixed.

If $X$ and $K$ are topological spaces, let $m(X ; K)$ be the following subspace of $K$,

$$
m(X ; K)=\{f(a X) \mid f \text { embeds } c X \text { in } K\} .
$$

Received by the editors April 14, 1966.

(1) This research was supported in part by NSF Grant 3894. 
If $g: Y \rightarrow X, f: c X \rightarrow K$ and $h: K \rightarrow L$ are embeddings, then the composite $h \circ f \circ c g$ is an embedding. Thus if $f(a X) \in m(X ; K)$, then the formula

$$
m(g ; h)(a X)=h f(c g(a Y)) \in m(Y ; L)
$$

defines an embedding

$$
m(g ; h): m(X ; K) \rightarrow m(Y ; L) .
$$

Thus $m(;)$ is seen to be a functor from embeddings to embeddings. It is contravariant in the first variable and covariant in the second. If $X_{0} \subset X$ and $K_{0} \subset K$, define

$$
m\left(X, X_{0} ; K, K_{0}\right)=\left(m\left(X_{0} ; K\right), m\left(X_{0} ; K_{0}\right) \cup m(X, K)\right)
$$

and

$$
m\left(X ; K, K_{0}\right)=\left(m(X ; K), m\left(X ; K_{0}\right)\right) .
$$

If $\phi$ is the empty space, then $m\left(X, X_{0} ; K, \phi\right)=\left(m\left(X_{0} ; K\right), m(X ; K)\right)$, which we denote by $m\left(X, X_{0} ; K\right)$. This notation agrees well with the observation that $m(X ; K, \phi)$ $=(m(X ; K), \phi)$. On the other hand, $m\left(X, \phi ; K, K_{0}\right)=\left(K, K_{0}\right)$, and this is ordinarily quite different from $m\left(X ; K, K_{0}\right)$.

These functions extend in the usual fashion to a functor defined on embeddings $f: Y, Y_{0} \rightarrow X, X_{0}$ and $g: K, K_{0} \rightarrow L, L_{0}$, and having values in the category of embeddings of topological pairs.

Note that isotopies of $f$ and $g$ induce isotopies of $m(f ; g)$. Since an isotopy is also a homotopy, the composite of $m$ with one of the standard functors of algebraic topology yields an isotopy functor with values in a category of homomorphisms of graded groups or rings. In the present paper, we choose homology theory for this purpose and study the various functors obtained by holding one of the two variables in $m$ fixed. Regardless of which variable is held fixed, the composite functor has an associated natural transformation (the boundary homomorphism), has the property that each topological pair leads to an exact sequence, and assigns the same homomorphisms to isotopic embeddings. In short, such a functor satisfies all the Eilenberg-Steenrod Axioms for a homology theory, save that the Excision and Dimension Axioms may fail and the Homotopy Axiom must be modified to become an Isotopy Axiom. Note that if the Excision Axiom is satisfied, such a functor is an isology functor [1].

2. Holding the second variable fixed. Select a topological pair $\left(K, K_{0}\right)$ and a homology theory $\left\{H_{n}\right\}$ with coefficients in an abelian group $G$, and consider the two contravariant functors,

$$
R(\quad)=m\left(; K, K_{0}\right) \quad \text { and } \quad T_{n}=H_{n} \circ R .
$$

Both are defined on some category of embeddings of topological pairs. The first has its values in the category of inclusions among pairs of subspaces of $K$ and the second has its values in the category of homomorphisms of graded abelian groups. 
It is almost immediate that if $f: Y \rightarrow X$ is an embedding, then $R(f): R(X) \rightarrow R(Y)$ is the inclusion map. It follows that any two maps $f, g: Y, Y_{0} \rightarrow X, X_{0}$ have the same image $R(f)=R(g)$. Thus the functor $R$ does not carry much information, nor can $T_{n}$.

In addition, $T_{n}$ need not be excisive, as the following example shows. Let $X$ be the 1-dimensional polyhedron sketched below.

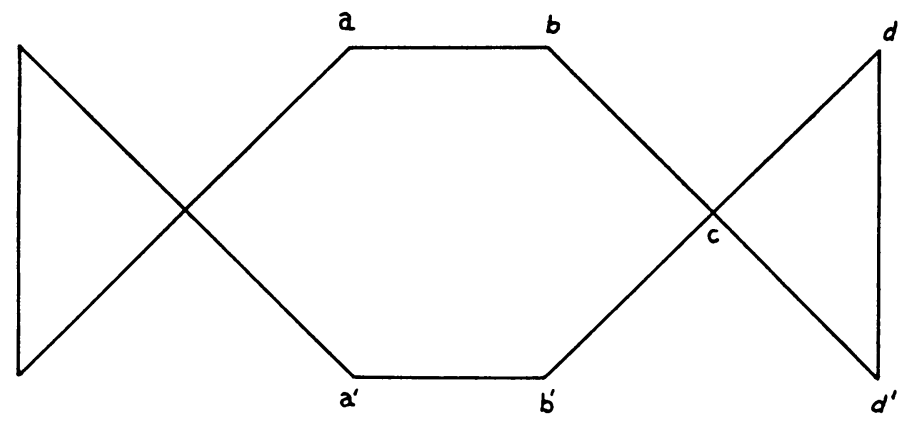

Let $\bar{U}$ be the subcomplex of $X$ consisting of the 1-simplexes spanned by $b, b^{\prime}$, $c, d$, and $d^{\prime}$. The open set $U$ is now obtained by removing the vertices $b$ and $b^{\prime}$ from $\bar{U}$. Let $A$ be the union of $\bar{U}$ with the 1-simplexes $a b$ and $a^{\prime} b^{\prime}$. Let $K=c(c 4)$ (the second cone over a set of four elements) and let $K_{0}$ be the empty subspace. Note that $K$ may be regarded as the union of four 2-simplexes $\sigma_{1}, \sigma_{2}, \sigma_{3}, \sigma_{4}$ sharing a 1-simplex $\tau=\sigma_{i} \cap \sigma_{j}(i \neq j)$. It is easily seen that $R(X)$ is empty, $R(A)=R(X-U)$ is the 1-simplex $\tau$ less its endpoints and $R(A-U)=K$. Thus $T_{0}(X, A)=G$ while $T_{0}(X-U, A-U)=0$.

I do not know whether there are any nontrivial excisive theories of this type.

3. Holding the first variable fixed. A family of isology functors is constructed in this section. To this end, select a paracompact space $X$, and use singular homology theory with coefficients in some abelian group $G$. Let $\bar{\Psi}$ be a category of embeddings of topological pairs. Define on $\bar{\Psi}$ the functors,

$$
R(\quad)=m(X ;) \quad \text { and } \quad T_{n}(\quad)=H_{n}(R(\quad) ; G) .
$$

The first of these has its values in the category of continuous functions (embeddings, in fact) between topological pairs, and the second in the category of homomorphisms of graded abelian groups. Associated with $T=\left\{T_{n}\right\}$ is a natural transformation $\partial$ (derived from the boundary homomorphisms of the homology theory) such that the diagram

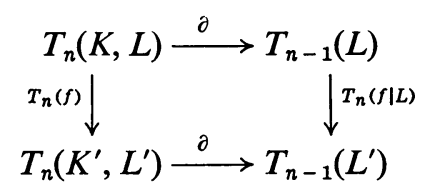

is commutative whenever $f: K, L \rightarrow K^{\prime}, L^{\prime}$ is in $\bar{\Psi}$. 
THEOREM 3.1. T, $\partial$ is an isology theory.

This theorem yields the following result immediately.

Corollary 3.2. If $f: K, L \rightarrow K^{\prime}, L^{\prime}$ is an isotopy equivalence, then $H_{*}\left(m\left(X, X_{0} ; f\right)\right)$ is an isomorphism for all pairs $\left(X, X_{0}\right)$.

The proof of Theorem 3.1 is simply a matter of checking the six axioms, and all of these verifications are easy, except that of the Excision Axiom. Lemma 3.4 shows that a strong form of this axiom holds.

LEMMA 3.3. If $X$ is a paracompact space, if $K$ is a topological space and if $V$ is open in $K$, then $m(X ; V)=m(X ; K) \cap V$.

Proof. Clearly $m(X ; K) \cap V \supset m(X ; V)$. Suppose $y \in m(X ; K) \cap V$. Then there exists an embedding $f: c X \rightarrow K$ with $f(a X)=y$. Let $q: X \times I \rightarrow c X$ be a quotient map which is one-to-one on $X \times(0,1]$ and has $q[X \times 0]=a X$. Since $(f q)^{-1}[V]$ is a neighborhood of $X \times 0$, there are an open cover $\mathfrak{U}$ of $X$ and numbers $e(U) \in(0,1)$ with $U \times[0, e(U)] \subset(f q)^{-1}[V]$ for each $U \in \mathfrak{u}$. Let $\left\{P_{u}: X \rightarrow[0,1]\right\}$ be a partition of unity subordinate to $\mathfrak{u}$. Define

$$
g(X)=\sum_{U \in \mathfrak{U}} P_{U}^{(x) e(U)} \quad \text { for } x \in X .
$$

Set $h_{0}(x, t)=(x, g(x) t)$ for $t \in I$ and check that the formula,

$$
h(z)=q h_{0}\left(q^{-1}(z)\right) \quad \text { for } z \in c X,
$$

defines an embedding $h: c X \rightarrow f^{-1}[V]$. Thus $f h$ embeds $c X$ in $V$ and

$$
y=f h(a X) \in m(X ; V) .
$$

LEMMA 3.4. Let $(K, L)$ be an object in $\bar{\Psi}$ and let $M$ be a subset whose closure $\bar{M}$ is contained in the interior $U$ of $L$. If the inclusion map

$$
i:(K-M, L-M) \rightarrow(K, L)
$$

is in $\bar{\Psi}$, then $T(i)$ is an isomorphism.

Proof. Let $M_{1}=m(X ; K) \cap \bar{M}$ and $M_{2}=m(X ; K-M) \cap \bar{M}$. Then

$$
M_{2} \subset m(X ; K-M) \cap U \subset \text { interior }(m(X ; L-M))
$$

and

$$
M_{1} \subset \text { interior }(m(X ; L)) .
$$

Thus the inclusion maps

$$
i_{1}:\left(m(X ; K)-M_{1}, m(X ; L)-M_{1}\right) \rightarrow m(X ; K, L)
$$

and

$$
i_{2}:\left(m(X ; K-M)-M_{2}, m(X ; L-M)-M_{2}\right) \rightarrow m(X ; K-M, L-M)
$$


induce isomorphisms in homology. But

$$
\begin{aligned}
m(X ; L)-M_{1} & =m(X ; L) \cap(L-\bar{M}) \\
& =m(X ; L-\bar{M}) \\
& =m(X ; L-M)-M_{2}
\end{aligned}
$$

and

$$
m(X ; K)-M_{1}=m(X ; K-M)-M_{2} .
$$

Thus the result follows from the fact that $i_{1}=R(i) i_{2}$.

For an application of these functors, let $X_{n}$ be a discrete space with $n$ points ( $n=0,1,3,4, \ldots$; the value $n=2$ is deliberately omitted) and let $G_{0}, G_{1}, G_{3}, G_{4}, \ldots$ be abelian groups. Since a direct sum of isology theories is an isology theory,

$$
T_{p}^{\prime}(K, L)=\sum_{n \neq 2} H_{p}\left(m\left(X_{n} ; K, L\right) ; G_{n}\right)
$$

determines an isology theory. If $f: c X_{m} \rightarrow c X_{n}$ is an embedding with $m<n$, then

$$
\operatorname{Im}\left(T_{0}^{\prime}(f)\right) \subset T_{0}^{\prime}\left(c X_{n}\right)
$$

is a nontrivial proper subgroup, and it follows from [1, Theorem 8.2] that an embedding $g: K \rightarrow L$ of 2-dimensional finitely triangulable spaces is an isotopy equivalence if and only if $T_{p}^{\prime}(g)$ is an isomorphism for all $p$.

If $X_{0}$ is a closed subspace of the paracompact space $X$, then the Excision Axiom holds for the functor

$$
(K, L) \rightarrow H_{*}\left(m\left(X, X_{0} ; K, L\right) ; G\right) .
$$

However, the Exactness Axiom may fail, as the following example shows. Let $X$ be the discrete 3-point space and let $X_{0}=\phi$. Suppose $K$ is the 1-dimensional polyhedron sketched below, and $L=\{a, b\}$.

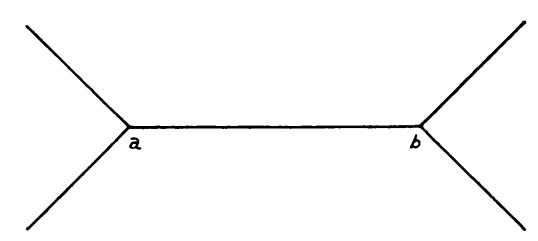

Then

$$
\begin{aligned}
m\left(X, X_{0} ; L\right) & =(L, \phi) \\
m\left(X, X_{0} ; K\right) & =(K, L)
\end{aligned}
$$

and

$$
m\left(X, X_{0} ; K, L\right)=(K, L) .
$$

Thus exactness fails in the 0 -dimensional groups of the homology sequence. 
4. Two-dimensional cones. Statement of results. This section is a study of the functors $H_{*}(m(X ;))$ on the category $\Phi$ of embeddings $f: c K \rightarrow c L$, where $K$ and $L$ are 1-dimensional, finitely triangulable spaces. Although these functors are not sufficiently discriminating to provide a classification by isotopy types, they come reasonably close. This statement is made precise by Theorem 4.1 and some examples.

The homology theory of this section is singular theory with integral coefficients.

THEOREM 4.1. If $f: c K \rightarrow c L$ is an embedding in $\Phi$ such that $H_{*}(m(X: f))$ is an isomorphism for all 1-dimensional finitely triangulable spaces $X$, then there exist closed subsets $K_{1} \subset K$ and $L_{1} \subset L$ such that

(1) $f\left[c K_{1}\right] \subset c L_{1}$,

(2) the restriction of $f$ to $c K_{1} \rightarrow c L_{1}$ is an isotopy equivalence, and

(3) $K-K_{1}$ and $L-L_{1}$ are finite unions of isolated arcs and points.

The proof of this theorem will be given in $\S 6$. The rest of the present section is given over to examples showing the theorem to be a best possible result.

Let $K_{1}=L_{1}$ be any 1-dimensional finitely triangulable space with a vertex of order 1, and let $A, B$ be finite discrete spaces disjoint from $K_{1}$. Set $K=K_{1} \cup A$ and $L=L_{1} \cup B$. In general, $c K$ and $c L$ have different isotopy types [2, Theorem 2.6].

Proposition 4.2. There is an embedding $f_{0}: K \rightarrow L$, and every embedding $f=c f_{0}: c K \rightarrow c L$ induces isomorphisms $H_{*}(m(X ; f))$ for all spaces $X$.

Proof. The space $A$ can be embedded in an arc. Since $L_{1}$ has a vertex $v$ of order 1 situated, let us say, on a 1-cell $\sigma$, there is an isotopy of the identity map on $L_{1}$ which shrinks $\sigma$ away from $v$ and leaves the rest of $L_{1}$ pointwise fixed. In this way an arc in $L_{1}$ is exposed, and $f_{0}$ is constructed by embedding $A$ in this arc. Note that maps $g_{0}: L \rightarrow K$ may be similarly constructed.

Let $X$ be any space. If the apex $a K$ lies in $m(X ; c K)$, then $m(X ; c K)$ is contractible, for whenever $y \in m(X ; c K)$, the interval from $y$ to $a K$ must also lie in $m(X ; c K)$. A similar statement holds for $m(X ; c L)$. Furthermore, $a K \in m(X ; c K)$ iff $a L \in m(X ; c L)$ for $f=c f_{0}$ and $g=c g_{0}$ preserve apexes. Thus $m(X ; f)$ will be a homotopy equivalence in this case and $H_{*}(m(X ; f))$ will be an isomorphism.

On the other hand, suppose $a K \notin m(X ; c K)$. Then $m(X ; c K)=m\left(X ; c K_{1}\right)$, for otherwise $c A \cap m(X ; c K)$ contains an arc, whence $c X$ embeds in an arc. Since $K$ contains two distinct points, $c K$ contains an arc having $a K$ as an interior point, whence $a K \in m(X ; c K)$. Similarly, $m(X ; c L)=m\left(X ; c L_{1}\right)$ in this case. But $f$ induces an isotopy equivalence of $c K_{1}$ with $c L_{1}$, and the theorem follows from Corollary 3.2 .

Similar examples are obtained when $K_{1}=L_{1}$ contains a simple closed curve and $A, B$ both contain some isolated arcs.

5. Lemmas about 2-dimensional cones. Let $K, L$ be 1-dimensional finitely triangulated spaces. Let $f: c K \rightarrow c L$ and $g: c L \rightarrow c K$ be embeddings that preserve 
apexes. The isotopy classes of $f$ and $g$ contain embeddings which preserve bases [2, Lemma 2.2], so there is no loss of generality in assuming that $f[K] \subset L$ and $g[L] \subset K$. In addition, select cellular decompositions for $K$ and $L$, such that each decomposition has a minimal number of vertices.

Suppose $M=K$ or $L$. In a minimal decomposition, the vertices of order 2 appear in the isolated simple closed curves, and only there. There is exactly one such vertex for each such curve, and it may lie anywhere on the curve. Apart from this ambiguity, the topology of $M$ determines the minimal decomposition. Let $M^{\prime}$ denote the set of vertices of order $\geqq 3$ in $M$ and let $M^{\prime \prime}$ be the union of all the closed 1-cells whose boundaries consist of vertices of orders $\geqq 2$. Note that the closed star of $M^{\prime \prime}$, star $M^{\prime \prime}$, will include all of $M$ save the isolated arcs and points.

LEMMA 5.1. The embedding $f$ induces (by restriction) homeomorphisms of $K^{\prime}$ onto $L^{\prime}$, of $c K^{\prime}$ onto $c L^{\prime}$, of $K^{\prime \prime}$ onto $L^{\prime \prime}$ and of $c K^{\prime \prime}$ onto $c L^{\prime \prime}$.

Proof. It suffices to show that $f$ maps the given subset of $K$ onto the corresponding subset of $L$ in each case.

It follows from [2, Lemma 2.2] that $f\left[c K^{\prime}\right] \subset c L^{\prime}$ and $g\left[c L^{\prime}\right] \subset c K^{\prime}$, whence $f\left[K^{\prime}\right] \subset L^{\prime}$ and $g\left[L^{\prime}\right] \subset K^{\prime}$. The same lemma shows that if $v$ is a vertex in $K$ of order $o(v) \geqq 3$, then $f(v)$ has order $o(f(v)) \geqq o(v)$. Also, $o(g f(v)) \geqq o(f(v))$. But $g f\left[K^{\prime}\right] \subset K^{\prime}$, whence, summing over $v \in K^{\prime}$,

$$
\sum o(v) \geqq \sum o(g f(v))
$$

Thus the inequalities must all be equalities: $o(v)=o(f(v))=o(g f(v))$, and $g f\left[K^{\prime}\right]=K^{\prime}$. A similar argument applies to $L^{\prime}$, so $f\left[K^{\prime}\right]=L^{\prime}$ and $g\left[L^{\prime}\right]=K^{\prime}$. Since $f[c v] \subset c(f(v))$ for each $v \in K^{\prime}$ and since the endpoints $v$ and $a K$ of the arc $c v$ are mapped onto the endpoints $f(v)$ and $a L_{0}$ of the arc $c(f(v))$, it follows that $f[c v]=c(f(v))$ whence $f\left[c K^{\prime}\right]=c L^{\prime}$. Similarly $g\left[c L^{\prime}\right]=c K^{\prime}$.

Let $\sigma$ be a 1-cell in $K^{\prime \prime}$, and assume that the boundary of $\sigma$ consists of two vertices $v, w \in K^{\prime}$. Since sufficiently small neighborhoods of $v$ map homeomorphically onto neighborhoods of $f(v)$, there must be a 1-cell $\tau$ of $L$ such that $f[\sigma]$ and $\tau$ agree, at least in some neighborhood of $f(v)$. The two arcs $f[\sigma]$ and $\tau$ will continue to agree until one of them terminates. If $f[w] \in \tau$, then $f(w)$ is a vertex of $\tau$ (for $f\left[K^{\prime}\right]=L^{\prime}$ ), whence $f[\sigma]=\tau$. If $u \neq f(v)$ is the other vertex of $\tau$ and $u \in f[\sigma]$, then the minimality assumption on the decomposition of $L_{0}$ insures that $u$ does not have order 2. Clearly $u$ does not have order 1 , either. Thus $u \in L^{\prime}=f\left[K^{\prime}\right]$, whence $u=f(w)$ and $\tau=f[\sigma]$ again. A similar argument shows that a 1-cell which begins and terminates in the same vertex $v \in K^{\prime}$ maps onto a 1-cell of the same type as itself. Now, if $\alpha$ is an isolated simple closed curve in $K$, then $\alpha \cap K^{\prime}$ is empty, whence $f[\alpha] \cap L^{\prime}$ is empty. Since $\alpha$ can not be embedded in an arc or a point, $f[\alpha]$ must be an isolated simple closed curve of $L_{0}$. Thus $f\left[K^{\prime \prime}\right] \subset L^{\prime \prime}$. Similarly $g\left[L^{\prime \prime}\right] \subset K^{\prime \prime}$. A simple counting argument now shows that these set inclusions are equalities. 
Again, if $\sigma$ is a 1-cell with boundary $\partial \sigma=\{v, w\} \subset K_{0}^{\prime}$, then the boundary of the 2-cell $c \sigma$ is $\partial \sigma=\sigma \cup c v \cup c w$, and the boundary of $c f[\sigma]$ is $f[\sigma] \cup c f(v) \cup c f(w)$. Since $f$ maps $\partial(c \sigma)$ onto $\partial(c f[\sigma])$ with degree 1 , is one-to-one, and maps the points of $c \sigma$ near to interior points of $\sigma$ onto points of $c f[\sigma]$ near to interior points of $f[\sigma]$, it follows that $f[c \sigma]=c f[\sigma]$. If $\partial \sigma$ is a single point, then $\partial c \sigma=\sigma$, and once more $f[c \sigma]=c f[\sigma]$. Thus $f\left[c K^{\prime \prime}\right]=c L^{\prime \prime}$.

Lemma 5.2. The embedding $f$ carries $c\left(\right.$ Star $\left.K^{\prime \prime}\right)$ into $c\left(\right.$ Star $\left.L^{\prime \prime}\right)$ and induces an isotopy equivalence between these subspaces.

Proof. Let $\sigma$ be a 1-cell which lies in Star $K^{\prime \prime}$, but not in $K^{\prime \prime}$. Thus one of its endpoint vertices is of order $\geqq 3$ and the other is of order 1 . Name these vertices $v$ and $w$ with $o(v) \geqq 3$ and $o(w)=1$. As before, there must be a 1-cell $\tau$ of $L$ such that $f[\sigma] \subset \tau$. One of the vertices of $\tau$ is $f[v]$ and, since $L^{\prime \prime} \subset f\left[K^{\prime \prime}\right]$, the other has order 1 . But $c v$ separates the space $c K$, and $c f(v)=f[c v]$ separates $c L$, whence $f[c \sigma] \subset c \tau$. It is easy to construct an isotopy from $f \mid c \sigma$ to a homeomorphism of $c \sigma$ onto $c \tau$, and this isotopy may be required to leave $c v$ fixed. Combining these isotopies (one for each cell $\sigma$ of the type described) yields an isotopy from $f \mid c$ Star $K^{\prime \prime}$ to a homeomorphism of $c$ Star $K^{\prime \prime}$ onto $c \operatorname{Star} L^{\prime \prime}$.

Let $\boldsymbol{O}$ be a simple closed curve and let $\boldsymbol{Y}$ be a triod (that is, $\boldsymbol{Y}=c \mathbf{3}$ where $\mathbf{3}$ is a 3-point discrete space). Set

$$
\begin{array}{ll}
X_{1}=\boldsymbol{Y}+\boldsymbol{Y}+\boldsymbol{Y} & X_{2}=\boldsymbol{O}+\boldsymbol{O} \\
X_{3}=\boldsymbol{O}+\boldsymbol{Y} & X_{4}=\boldsymbol{O} \vee \boldsymbol{O}
\end{array}
$$

where + denotes disjoint union and $v$ is the union with exactly one point in common. If $P$ is a space, and $x_{0}, x_{1}$ points not in $P$, let $s P=P_{*}\left\{x_{0}, x_{1}\right\}$ be the suspension of $P$.

LeMma 5.3. An object $c K$ in $\Phi$ can be embedded in a book iff $m\left(X_{i} ; c K\right)$ is empty for $i=1,2,3,4$.

Proof. Observe that a book with $n$ leaves is homeomorphic with $c s F$ when $F$ is a discrete space with $n$ points. A shrinking towards the apex yields an embedding $c s F \rightarrow c s F-s F$ isotopic to the identity. It follows easily that any embedding $c X \rightarrow c s F$ is isotopic to one sending $X$ into $s F$.

Suppose $c K$ embeds in $c s F$. If $m\left(X_{i} ; c K\right)$ were nonempty, then there would be an embedding of $X_{i}$ in $s F$, and a routine check shows that this is impossible.

Conversely, suppose $m\left(X_{i} ; c K\right)$ is empty for $i=1,2,3,4$. Select a minimal cellular decomposition for $K$. Since $m\left(X_{1} ; c K\right)$ is empty, $K$ has at most 2 vertices of order $\leqq 3$. If $K$ has no vertices of order $\geqq 3$, then it consists of isolated points, arcs, and simple closed curves. Since $m\left(X_{2} ; c K\right)$ is empty, there is at most one simple closed curve. An embedding $f: K \rightarrow s F$ is easily constructed when $F$ has 3 points, and $c f$ is the desired embedding of $c K$ in a book. If $K$ has exactly one vertex $v$ of 
order $n \geqq 3$, then it has at most one simple closed curve and this contains $v$ (for $m\left(X_{3} ; c K\right)$ and $m\left(X_{4} ; c K\right)$ are empty). Let $F$ be a discrete space with $n+1$ points, and construct $f: K \rightarrow s F$ as follows. Let $w_{0}, w_{1}$ be the two vertices of order $n+1$ in $s F$, and set $f(v)=w_{0}$. If $\sigma$ is a 1-cell which begins and terminates at $v$, then $f(\sigma)$ runs along one arc from $w_{0}$ to $w_{1}$ and back along another. The other 1-cells radiating from $v$ are embedded in some of the remaining 1-cells of $s F$ so that their images start at $w_{0}$ but do not run all the way to $w_{1}$. The remainder of $K$ consists of isolated arcs and points; these are embedded in the $(n+1)$ st 1-cell of $s F$. Again $s f$ embeds $c K$ in the book $c s F$. The case in which $K$ has two vertices of orders $m \geqq n \geqq 3$ is similarly treated. In this case $c K$ is embedded in a book with $m+1$ leaves.

Given integers $p, q, r$, with $0 \leqq q \leqq r$ and $p \geqq 2$, there is a connected 1-dimensional finitely triangulated space $N(p, q, r)$ with exactly one vertex of order $p$, one of order $r, q$ arcs running between these two, and $p+r-2 q$ vertices of order 1 . Of these last mentioned vertices, $p-q$ terminate arcs radiating from the vertex of order $p$ and $r-q$ terminate arcs radiating from the vertex of order $r$. Note that the topological type of $N(p, q, r)$ is determined by this description.

Lemma 5.4. An object $c K$ of dimension 2 in $\Phi$ can be embedded in a book iff there are integers $p, q, r(q \leqq p \leqq r$ and $p \geqq 2)$ such that

$$
K=N(p, q, r) \cup A
$$

where $A$ is a finite union of points and arcs disjoint from $N(p, q, r)$ and each other.

Proof. Let $c(c F)$ be the book, where $F$ is a finite set. The spine of the book is the arc $c(a F)$ running between the apexes $a(c F)$ and $a F$; the leaves are the components of the complement of the spine. Every point in a book may be regarded as an apex over a 1-dimensional space $L$ : use $L=c F$ for the ends of the spine; $L=s F$ for interior points of a leaf and $L$ is an arc for edge points of a leaf. Thus the proof of the lemma is reduced to checking which spaces $K$ may be embedded in $L$. The possibility that $K$ might be a finite space is eliminated by the requirement that $c K$ have dimension 2.

Lemma 5.5. Let $M=N(p, q, r) \cup A$, where $A$ is a union of $n$ arcs disjoint from $N(p, q, r)$ and from each other. If $s>2$, then the number of components of $m(N(s, s, s), c M)$ is

$$
\begin{aligned}
& 1 \text { if } s \leqq q, \\
& 2 \text { if } q<s \leqq p, \\
& 1 \text { if } p<s \leqq r,
\end{aligned}
$$

and

$$
0 \text { if } r<s \text {. }
$$


If $s=2$, the number of components is

$$
\begin{aligned}
& 2+n \text { if } q=0 \\
& 1+n \text { if } q=1
\end{aligned}
$$

and

$$
1 \text { if } q>1 \text {. }
$$

Proof. Let $v$ and $w$ be the two distinguished vertices of $N(p, q, r)$ of orders $p$ and $r$, respectively, and let $M^{*}=m(N(s, s, s), c M)$. Suppose $s>2$, whence $M^{*}$ $=m(N(s, s, s), c N(p, q, r))$. If $s \leqq q$, then $M^{*}$ consists of the apex together with the interior points of the two spines, i.e., $M^{*}=(c v) \cup(c w)-\{v, w\}$. This follows at once from [2, Lemma 2.1]. If $q<s \leqq p$, then the apex $a=a N(p, q, r)$ is not in $M^{*}$, and so $M^{*}=(c v) \cup(c w)-\{v, w, a\}$ has two components. If $p<s \leqq r$, then $M^{*}$ $=c w-\{w, a\}$ has one component. Clearly $M^{*}$ is empty when $s>r$.

If $s=2$, then $M^{*}$ consists of all points which can be surrounded by a disc in $c M$. If $q=0$, then $c N(p, q, r)$ is a union of two books having exactly one point, the apex, in common. Thus, $c M$ is the union of these two books with a collection of $n$ triangles, and the apex is the only point shared by any two of these summands, whence $M^{*}$ has $n+2$ components. The remaining two cases are similar.

6. Proof of Theorem 4.1. Assume that $f: c K \rightarrow c L$ induces isomorphisms $H_{*}(m(X: f))$ for all 1-dimensional finitely triangulable spaces $X$. Since $m(L ; c L)$ is nonempty and since $H_{0}(m(L ; f))$ is an isomorphism, $m(L ; c K)$ is nonempty; let $g: c L \rightarrow c K$ be an embedding.

The case $f(a K)=a L$ and $g(a L)=a K$. Let $K_{1}=$ Star $K^{\prime \prime}$ and $L_{1}=\operatorname{Star} L^{\prime \prime}$. Then Lemma 5.2 shows that $f$ restricts to an isotopy equivalence of $c K_{1}$ with $c L_{1}$.

The cases $f(a K) \neq a L$ or $g(a L) \neq a K$. In these cases the proof reduces to showing that $K_{1}$ and $L_{1}$ may be selected such that $c K_{1}$ and $c L_{1}$ are books with the same number of leaves. Only the case $f(a K) \neq a L$ is treated; the case $g(a L) \neq a K$ is similar.

Every point of $c L$ except possibly $a L$ has a neighborhood that is a book. Thus, preceding $f$ by a radial shrinking of $c K$ towards $a K$ (if necessary), $c K$ is embedded in a book. It follows from Lemma 5.3 that $m\left(X_{i} ; c K\right)$ is empty for $i=1,2,3,4$. Since $H_{0}\left(m\left(X_{i} ; f\right)\right)$ is an isomorphism, $m\left(X_{i} ; c L\right)$ is empty for $i=1,2,3$, 4, whence $c L$ embeds in a book. Lemma 5.4 now shows that there are integers $p, q, r, u, v, w$, such that

$$
\begin{aligned}
& K=N(p, q, r)+A, \\
& L=N(u, v, w)+B,
\end{aligned}
$$

where $A, B$ are finite unions of isolated arcs and points, and + denotes disjoint union. If $X$ is any space, then the image of $m(X ; c K)$ under $f$ lies in a single component of $m(X ; c L)$, because $m(X ; C)$ is connected whenever $C$ is a book. This 
implies that $m(X ; c K)$ and $m(X ; c L)$ must be connected for all $X$. Thus it follows from Lemma 5.5 (with $X=N(2,2,2)$ ) that $q>0$ and $v>0$. These same observations, with $X=N(p, p, p)$, show that $p=q$ or $p=2$. The spaces $c N(p, q, r)$ with $(p, q)$ $=(2,1)$ or $p=q$ are all isotopic to books with $r$ leaves. Similarly $c N(u, v, w)$ is isotopic to a book with $w$ leaves. Applying Lemma 5.5 again, together with the fact that $H_{0}(m(N(s, s, s) ; f))$ is an isomorphism for $s=r, w$, shows that $r=w$. Since every embedding of a book with $r$ leaves into itself is an isotopy equivalence, the proof of the theorem in this case is completed by setting $K_{1}=N(p, q, r)$ and $L_{1}=N(u, v, w)$.

7. Deleted products. If $Y$ is a topological space, then the deleted product space $R Y=Y \times Y-\Delta Y$ is the topological product of $Y$ with itself minus the diagonal. This construction extends to a functor $R$ on embeddings of topological pairs in the obvious way. The composite of this functor with the integral singular homology functor $H_{*}$ is a functor with values in the category of homomorphisms of graded abelian groups. It is this functor that will almost fill the gap left by the functors $H_{*}(m(X ;))$.

Let $K=K_{1} \cup A$ and $L=L_{1} \cup B$ be finitely triangulable 1-dimensional spaces in which $A$ and $B$ are finite unions of isolated arcs and points. Let $f=c K \rightarrow c L$ be an embedding such that $f\left[c K_{1}\right] \subset c L_{1}$ and such that the restriction $c K_{1} \rightarrow c L_{1}$ is an isotopy equivalence.

THEOREM 7.1. If $H_{*}(R(f))$ is an isomorphism and if neither $K_{1}$ nor $L_{1}$ is contractible, then $f$ is an isotopy equivalence.

Proof. First suppose $f$ preserves apexes. Then there is no loss of generality in assuming $f[K] \subset L$ and $f\left[K_{1}\right] \subset L_{1}$. This part of the proof consists in using [2, Propositions 3.2-3.5] to show that the restriction $f_{0}: K \rightarrow L$ of $f$ is an isotopy equivalence. Now, no isolated point or arc $A_{1}$ of $A$ maps into $L_{1}$, for if it did, then $f_{0}$ could be factored

$$
K \stackrel{g}{\longrightarrow} K \cup C \stackrel{h}{\longrightarrow} L
$$

where $C$ is an arc from $A_{1}$ to a vertex $v$ of $K$. But Lemma 5.1 shows that $v$ must be of order 1. Thus if $\beta_{1}$ is the $i$ th Betti number of $K$, then the kernel of $H_{n}(R(c g))$ would have rank $2 \beta_{1}$ for $n=2$ and $2 \beta_{0}-4$ or $2 \beta_{0}-2$ for $n=1$ (the latter depending on whether $A_{1}$ is a point or an arc). Since $K_{1}$ is not contractible, these ranks could not both be zero, whence $H_{*}(R(f))$ could not be an isomorphism. Similar arguments show that $f_{0}[K]$ meets each component of $L$, that no isolated point of $A$ maps into an arc of $B$ and that no two arcs of $A$ map into the same arc of $B$.

Now suppose $f(a K) \neq a L$. Then $f$ may be factored through a book,

$$
c K \underset{g}{\longrightarrow} c(c F) \underset{h}{\longrightarrow} c L
$$

where $F$ is a finite discrete space, $c F \subset L_{0}$, and $h$ is the inclusion map. Since $c F$ is connected and $c F \subset L_{1}$, it is easily demonstrated that $K_{1}$ has the isotopy type of 
$c F$. Thus $c K \rightarrow c(c F) \rightarrow c L_{1}$ is an isotopy equivalence. But the case of $L_{1}$ being contractible was excluded and $c L_{1} \rightarrow c L$ preserves apexes, so the argument in the first half of this proof shows that $c L_{1} \rightarrow c L$ is an isotopy equivalence, whence $f$ is.

Now let us ask what nonequivalences $f$ in $\Phi$ induce isomorphisms under all of the functors we have considered. These exceptional cases are of the following two types.

(1) Let $K$ be a finitely triangulable 1-dimensional contractible space with at least two vertices of orders $\geqq 3$, let $L$ be the union of $K$ with an isolated point, and let $f: c K \rightarrow c L$ be the inclusion.

(2) Let $L$ be a finitely triangulable 1-dimensional contractible space with at least two vertices of orders $\geqq 3$ and let $K=L \cup A$ be the union of $L$ with an isolated point $A$. Since $L$ is contractible, it has a vertex $v$ of order 1 and a shrinking $f_{1}: L \rightarrow L$ such that $v \notin f_{1}[L]$.

Let $f_{0}: K \rightarrow L$ agree with $f_{1}$ on $L$ and send $A$ to $v$. Set $f=c f_{0}$.

Other examples of these types are obtained by varying $L$ and $f$ by isotopies. The proofs that $H_{*}(m(X ; f))$ and $H_{*}(R(f))$ are isomorphisms are routine. The requirement that $K$ and $L$ have at least two vertices of orders $\geqq 3$ is needed, for otherwise $c K$ and $c L$ have the same isotopy type, namely that of a book.

\section{REFERENCES}

1. A. H. Copeland, Jr., Isology theory, Trans. Amer. Math. Soc. 107 (1963), 197-216.

2. - Isotopy of 2-dimensional cones, Canad. J. Math. 18 (1966), 201-210.

NORTHWESTERN UNIVERSITY,

Evanston, IllinoIs 